\title{
Why and How Often Is Revision Surgery Necessary after First Metatarsophalangeal Joint Arthrodeses? A Cohort of 120 Consecutive Cases
}

\author{
Lisca Drittenbass ${ }^{1}$, Halah Kutaish ${ }^{1,2 *}$, Leow Voon Chin ${ }^{1}$, Richard Stern ${ }^{1}$, Mathieu Assal ${ }^{1,2}$ \\ ${ }^{1}$ Centre for Surgery of the Foot \& Ankle, Hirslanden Clinique La Colline, Geneva, Switzerland \\ ${ }^{2}$ Faculty of Medicine, Geneva University, Geneva, Switzerland \\ Email: *hala.kutaish@gmail.com
}

How to cite this paper: Drittenbass, L., Kutaish, H., Chin, L.V., Stern, R. and Assal, M. (2021) Why and How Often Is Revision Surgery Necessary after First Metatarsophalangeal Joint Arthrodeses? A Cohort of 120 Consecutive Cases. Open Journal of Orthopedics, 11, 221-232.

https://doi.org/10.4236/ojo.2021.118021

Received: May 20, 2021

Accepted: August 9, 2021

Published: August 12, 2021

Copyright $\odot 2021$ by author(s) and Scientific Research Publishing Inc. This work is licensed under the Creative Commons Attribution International License (CC BY 4.0).

http://creativecommons.org/licenses/by/4.0/

\begin{abstract}
Background: Little is known about the rate and reasons for revision after primary Metatarsophalangeal (MTP1) arthrodesis with the latest implants. While it is commonly assumed that nonunion accounts for most reoperations, we hypothesized that malposition is the leading cause of revision. The aim of this study was to determine the rate and reasons for revision after MTP1 arthrodesis using cup- and cone-reamers and the latest locking plate technology. Method: Between 2015 and 2017, 120 consecutive MTP1 fusions in 114 patients were performed with a low profile, pre-contoured titanium dorsal locking plate and a plantar metatarsophalangeal screw. The rate and reasons for revision within a minimum one-year follow-up period (average 16 months [12 - 26]) after index procedures were documented and analyzed. The revision was defined as any reoperation following the index procedure excluding hardware removal. Results: Seventeen of 120 feet (14\%) underwent revision. Four patients developed a nonunion (3.3\%) and needed revision, and 11 feet (9\%) required revision for painful malposition; insufficient extension and excessive valgus accounted for the majority. Two patients required medial sesamoidectomy. No infection or wound healing problems occurred. Twelve patients requested hardware removal. Conclusions: Nonunion revision rate after MTP1 fusion with the latest locking plate technology is low. The leading cause of revision is malposition followed by nonunion. Excessive valgus and insufficient extension account for most symptomatic malposition. A recommendation of 0 - 10 degrees of valgus in the horizontal plane may contribute to diminishing revision rates; no conclusions could be drawn regarding an optimal position in the sagittal plane.
\end{abstract}




\section{Keywords}

Metatarsophalangeal Joint Fusion, MTP1 Arthrodesis, MTP1 Fusion, Malposition, Reoperation, Revision

\section{Introduction}

First Metatarsophalangeal (MTP1) joint arthrodesis is a commonly performed procedure for the treatment of hallux rigidus, severe and recurrent bunion deformities, rheumatoid arthritis, and other less common disorders of the MTP1 joint. Results have shown good outcomes with high patient satisfaction [1].

A variety of fixation techniques and implants have been described over the past decades, including Kirschner wires, memory staples, one or two metatarsophalangeal screws in a crossed or parallel manner, as well as a variety of plates. All these methods aim to achieve a stable fixation to maintain the desired position of fusion and allow for uneventful bone healing. Of the different fixation methods, the use of a dorsal locking plate with a plantar metatarsophalangeal screw has been shown to be biomechanically superior, [2] [3] [4] and is currently the most commonly used fixation technique. Current data suggests that this type of fixation has reduced the nonunion rate from $10 \%-20 \%$ to $2 \%-14 \%$ [1] [3] [5] [6].

With decreasing nonunion rates, the importance of the position of fusion for patient satisfaction becomes clearer [7]. Thus, efforts have been made to develop means to improve the precision of intraoperative positioning. Cup- and conereamers for dome-shaped joint preparation allow for meticulous positioning without excessive loss of metatarsal length. The previously used preparation technique with flat cuts leads to loss of length if re-cutting is necessary to change position and is therefore increasingly abandoned. Furthermore, superior stability and a larger contact area of dome-shaped joint preparation have been demonstrated in biomechanical studies [4].

To facilitate perfect positioning the latest generation of dorsal plates are pre-contoured and provide variable degrees of dorsiflexion and valgus. The pre-bent plate serves as a template for arthrodesis positioning [6]. These plates are low profile while still providing high stability, thus reducing soft tissue problems due to prominent hardware.

Although it is assumed that the need for reoperation after MTP1 fusion with current implants and preparation techniques is infrequent, little is known about the rate and the reasons for the revision. It is commonly presumed that nonunion accounts for most revisions [8]. Other perceived reasons are wound healing issues due to prominent hardware, malposition, degeneration of the sesamoid complex, and infection. We hypothesized that malposition is the leading cause of reoperation.

The purpose of this study was to determine the rate and reasons for reopera- 
tion after MTP1 fusion using the latest generation plate and a plantar metatarsophalangeal screw.

\section{Materials and Methods}

Between 2015 and 2017, 120 consecutive MTP1 fusions in 114 patients were included at Centre Assal, Clinique La Colline, Hirslanden, in this retrospective study. Fusion was performed with a low profile, precontoured, titanium dorsal locking plate (APTUS ${ }^{\circledR}$ Foot MTP Fusion plate, MEDARTIS ${ }^{\circledR}$, Basel, Switzerland) providing for a dorsiflexion angle of 0,5 , or 10 degrees, as well as 10 degrees of valgus. A plantar metatarsophalangeal screw was additionally used. Inclusion criteria were: 1 ) patients of at least 18 years of age at the time of surgery; 2) diagnosed with hallux rigidus, hallux valgo-rigidus and inflammatory arthritis; 3) primary MTP1 fusion with APTUS ${ }^{\circledR}$ MTP1 fusion plate performed from 01.01 .2015 - 30.04.2017; 4) surgical procedures performed by the study senior surgeons (MA and LD). While exclusion criteria were: 1) any patient under the age of 18 at the time of surgery; 2) any patient not operated on with APTUS ${ }^{\circledR}$ MTP1 fusion plate; 3) patients operated on before 01.01.2015 or after 30.04.2017, or 4) patients not operated by the two senior surgeons (MA and LD).

Basic patient demographic characteristics including age, sex and operated side were recorded, as well as indication for the index surgery (Table 1). Preoperative weight bearing radiographs were performed to confirm MTP1 joint degeneration and/or severe hallux valgus, the latter defined as an intermetatarsal angle (IMA = angle between the first and second metatarsal longitudinal axis in the horizontal plane) greater than 20 degrees or Hallux Valgus Angle (HVA) greater than 35 degrees.

Radiologic assessment was performed by two orthopaedic surgeons not involved in the surgery. Postoperative weight bearing radiographs were performed at six weeks and three months, as well as six and twelve months if union was not achieved earlier. Any radiographic findings such as loosening of the implant, hardware failure, sclerosis and lack of consolidation were noted. Suspected nonunion at nine to twelve months was confirmed by Computed Tomography (CT scan). The position of the MTP1 arthrodesis was assessed on weight bearing radiographs at six months. The dorsal extension of the proximal phalanx in the sagittal plane was measured in reference to the floor. The postoperative HVA

Table 1. Patient demographics.

\begin{tabular}{ccc}
\hline & $\begin{array}{c}\text { Not reoperated }(\mathbf{N}=91) \\
\text { Mean } \pm \text { SD }\end{array}$ & $\begin{array}{c}\text { Reoperated }(\mathbf{N}=29) \\
\text { Mean } \pm \text { SD }\end{array}$ \\
\hline Age (years) & $62 \pm 10$ & $61 \pm 11$ \\
\hline Sex (female) & $\mathbf{N}(\%)$ & $\mathbf{N}(\%)$ \\
Indication (HVR) & $82(90 \%)$ & $24(83 \%)$ \\
Side (right) & $76(83 \%)$ & $18(62 \%)$ \\
\hline
\end{tabular}


and IMA were measured.

Radiographic malposition of the MTP1 fusion was defined as follows: in the horizontal plane any varus, or more than 15 degrees of valgus; in the sagittal plane an angulation of the proximal phalanx in reference to the floor less than 5 or greater than 15 degrees of extension in feet without sagittal translation of the metatarsal head (elevatus). In feet presenting with metatarsus primus elevatus, 0 - 15 degrees of extension was accepted. Rotational positioning of the toe was not measured on radiographs but estimated clinically.

Postoperative follow-up visits were routinely performed at one, three and six weeks, and three, six and twelve months. The mean follow-up was 16 months (range, 12 - 26). Twelve patients were last seen at six months postoperative. They were asymptomatic with a radiographically and clinically healed MTP1 fusion and did not return for their twelve-month follow-up visit. It can only be assumed that they were doing well. Any complications such as delayed wound healing, infection, hypertrophic scarring, postoperative stress fractures, nonunion or pain at the sesamoid complex were noted.

The position of the MTP1 fusion was evaluated clinically in the weight bearing patient. Rotation of the toe (pronation/supination) was noted using the coronal plane of the nail in reference to the floor. Symptoms of malposition were documented, and included pain under MTP1, pain under the condyles of the first phalanx, pain on the apex of the first toe, pain over the Interphalangeal (IP) joint, nail dystrophy, recruitment of the flexor apparatus, and painful contact of the first and second toes. Malposition was defined as any position sufficiently symptomatic according to the above to necessitate surgical repositioning. All reoperations that were performed during the follow-up period were recorded. Revision was defined as any reoperation following the index procedure excluding hardware removal.

\subsection{Operative Technique}

Patients were placed supine with an ankle tourniquet inflated to $230 \mathrm{mmHg}$. A longitudinal dorsomedial incision was made to approach the MTP1 joint. The extensor hallucis longus was carefully retracted laterally in its sheath. The joint was exposed and a $1.6 \mathrm{~mm}$ Kirschner wire was drilled into the shaft along the axis of the first metatarsal from the center of the head. A cannulated cup-shaped reamer (APTUS ${ }^{\circledR}$ Foot, MEDARTIS ${ }^{\circledR}$, Basel, Switzerland) sized according to the metatarsal head was used to ream to well-vascularized subchondral bone. The proximal phalanx was prepared in the same fashion using the matching domeshaped reamer. After irrigation the surface of the articulation was further prepared by drilling multiple holes with a $2.3 \mathrm{~mm}$ drill bit. Dorsal osteophytes were meticulously removed with a small oscillating saw. According to patient foot type, a plate with 0,5 , or 10 degrees of dorsiflexion was chosen and preliminarily placed on the prepared MTP1 joint with Kirschner wires. This allowed use of the bend of the plate for positioning the arthrodesis. The position was checked by simulating weight bearing on a flat sterile teflon board, such that with the foot 
flat on the board the tip of the toe had to have light contact with it (Figure 1(a)). Performing a heel lift of $3 \mathrm{~cm}$ off the board should not cause extension of the IP joint (Figure 1(b)). Once the desired position was achieved, the plate was fixed using six locking screws, three distal and three proximal (Figure 2). Finally, an independent $2.5 \mathrm{~mm}$ metatarsophalangeal screw was placed on the plantar aspect of the joint from proximal-medial to distal-lateral in order to enhance stability. Final control of the position was performed using simulated weight bearing as mentioned above, as well as fluoroscopy. Following wound closure a soft compressive sterile dressing was applied.

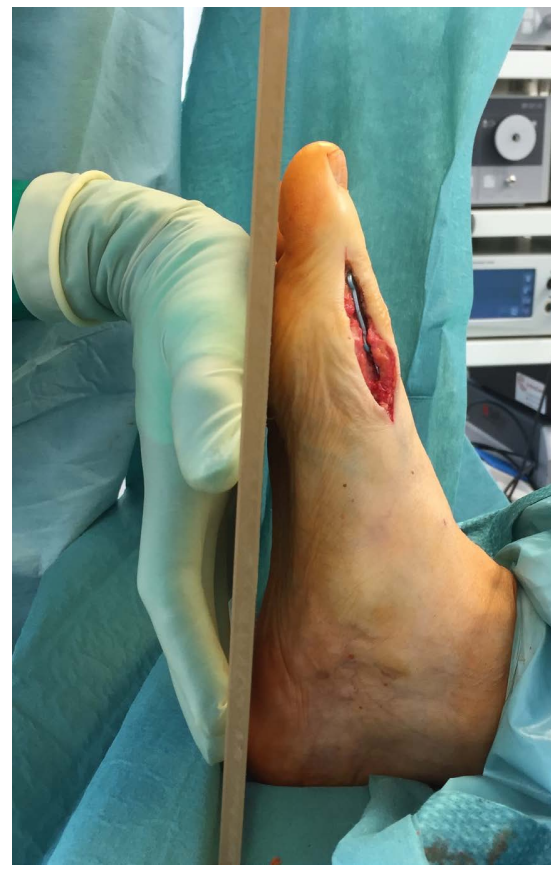

(a)

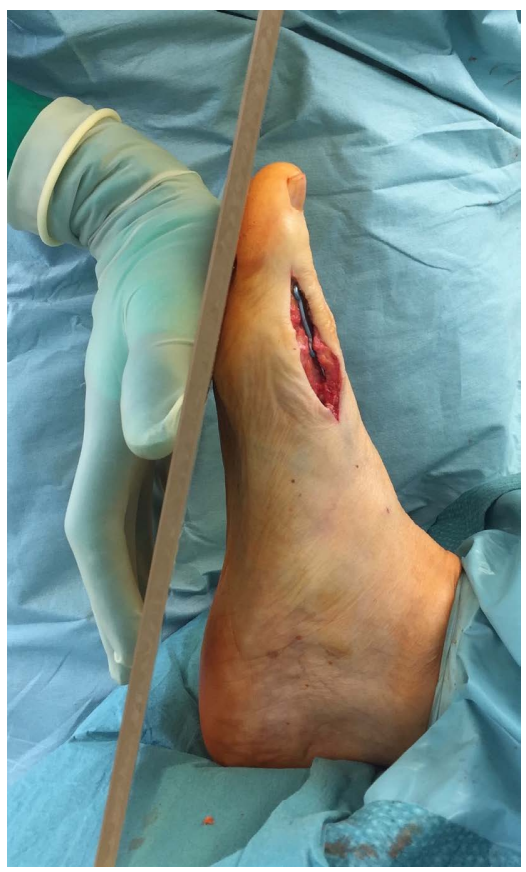

(b)

Figure 1. Intraoperative testing of the correct position of the first MTP1 joint fusion by simulating weight bearing on a flat board: (a) The tip of the toe had to touch the board lightly. (b) Performing a heel lift of three centimeters should not cause extension of the interphalangeal joint.

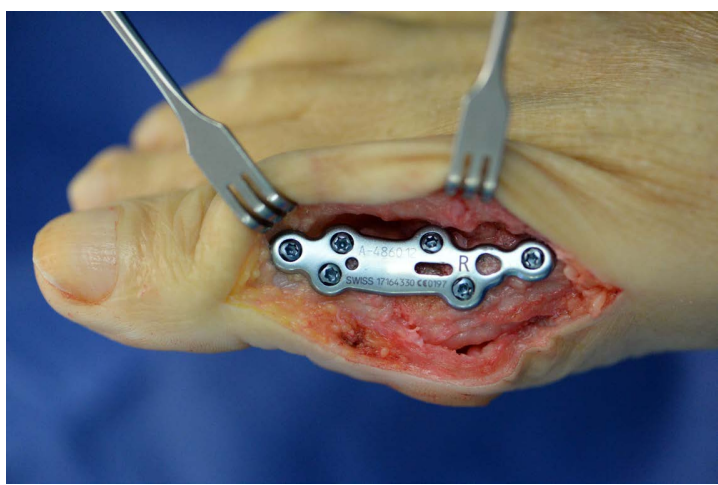

Figure 2. Fusion of the first MTP1 joint with a low profile, pre-contoured, titanium dorsal locking plate of the latest generation. In addition to the material visible on this figure, a plantar metatarsophalangeal screw was placed to enhance stability. 
Patients were allowed to be weight bearing as tolerated with a stiff-soled medical shoe for six weeks.

\subsection{Statistical Methods}

Exploratory data analysis was performed by means of scatterplot graphs.

\section{Results}

Seventeen of 120 feet (14\%) had to undergo revision. Of all patients, four suffered from a symptomatic nonunion (3.3\%) and were treated by revision surgery. No asymptomatic nonunions occurred. Of these four patients, three were initially operated upon for hallux valgo-rigidus, and one for hallux rigidus. One patient had undergone a one-stage bilateral fusion.

Eleven of 120 feet (9\%) underwent revision for malposition (Table 2). Malposition in the sagittal plane with insufficient extension accounted for four of the 11 malposition cases and was treated by a dorsiflexion, closing wedge osteotomy at the fusion site. Radiographically, dorsal extension of the MTP1 fusion in these four patients revealed a mean of 4.3 degrees (range, $0-8.5$ ). In the 120 index patients, the postoperative mean dorsal extension was of 4.6 degrees (range, 0 - 14 degrees) (Figure 3). Seventy-six patients presented with an extension of less than 5 degrees, and all these patients displayed metatarsus primus elevatus.

Five patients presented with malposition in the horizontal plane with painful contact of the first and second toes, and required correction with an Akin osteotomy

Table 2. Patients with malposition needing repositioning revision surgery (Diag $=$ Diagnosis $\mathrm{HR}=$ hallux rigidus, $\mathrm{HVR}=$ hallux valgo-rigidus, $\mathrm{HVA}=$ hallux valgus angle, IMA $=$ intermetatarsal angle, Ext $=$ extension, Rot $=$ rotation $)$.

\begin{tabular}{cccccccccc}
\hline Patient & Age & Sex & Diag & $\begin{array}{c}\text { Preop } \\
\text { HVA }\end{array}$ & $\begin{array}{c}\text { Postop } \\
\text { HVA }\end{array}$ & $\begin{array}{c}\text { Preop } \\
\text { IMA }\end{array}$ & $\begin{array}{c}\text { Postop } \\
\text { IMA }\end{array}$ & $\begin{array}{c}\text { Postop } \\
\text { Ext }\end{array}$ & $\begin{array}{c}\text { Type of } \\
\text { revision }\end{array}$ \\
\hline 1 & 71 & F & & & 10.7 & & 9.0 & 0.0 & Akin \\
2 & 72 & F & HVR & 26.7 & 20.7 & 11.7 & 10.2 & 3.1 & Akin \\
3 & 74 & F & HVR & 35.6 & 21.8 & 13.4 & 12.5 & 2.8 & Akin \\
4 & 43 & M & HR & 7.1 & 9.5 & 7.3 & 10.0 & 1.5 & Akin \\
5 & 39 & F & HVR & 28.1 & 10.2 & 15.6 & 6.4 & 3.1 & Akin \\
Mean & & & & 24.4 & 14.6 & 12.0 & 9.6 & 2.1 & \\
6 & 71 & F & HVR & 39.5 & 6.6 & 19.3 & 11.3 & 5.2 & Ext \\
7 & 72 & F & HVR & 24.0 & 6.3 & 12.4 & 8.2 & 8.5 & Ext \\
8 & 55 & F & HVR & 32.5 & 0.0 & 23.4 & 5.9 & 0.0 & Ext \\
9 & 61 & F & HV & 17.6 & 7.2 & 9.4 & 9.4 & 3.6 & Ext \\
Mean & & & & 28.4 & 5.0 & 16.1 & 8.7 & 4.3 & \\
10 & 42 & M & & & 16.3 & & 7.5 & 6.1 & Rot \\
11 & 73 & F & HVR & 36.6 & 16.2 & 12.7 & 6.8 & 10.6 & Rot \\
Mean & & & & 36.6 & 16.2 & 12.7 & 7.1 & 8.3 & \\
\hline
\end{tabular}




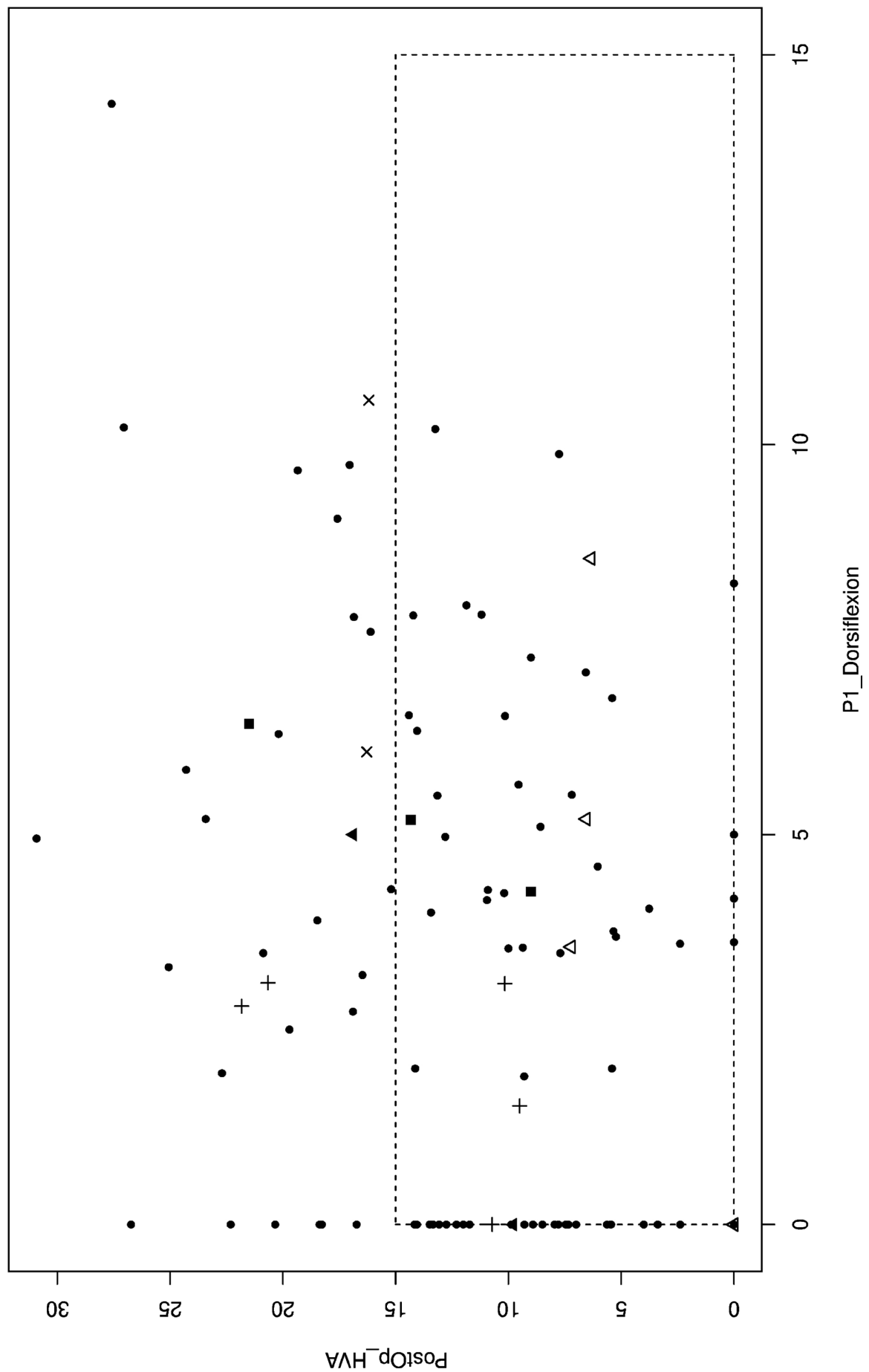

Figure 3. The graph shows the position of the first MTP1 joint for all patients in the horizontal (postop_HVA) and sagittal (P1_Dorsiflexion) plane. Dorsiflexion of the toe was measured in reference to the floor. The dotted rectangle represents the recommended range of position. ( $\bullet$ no reoperation needed, - nonunion, $\boldsymbol{\Delta}$ sesamoidectomy, x derotation, + extension osteotomy, $\Delta$ Akin osteotomy).

(medial closing wedge varisation osteotomy at the base of first phalanx). Radiographically those five patients showed a mean preoperative HVA of 24.4 degrees (range, 7 - 36) and a mean postoperative HVA of 14.5 degrees (range, 10 - 21); 
only two of them showed actual radiological malposition with a HVA greater than 15 degrees (Figure 3 ). The preoperative IMA in this group measured a mean 14.6 degrees (range, 9.5 - 21.8) and was corrected to within normal range postoperatively (mean 9 degrees; range, 6 - 12). In the 120 index patients, the postoperative position in the horizontal plane was found to be a mean valgus of 12 degrees (range, 0 - 31) (Figure 3). It had been corrected from a preoperative mean of 26 degrees (range, 2 - 52). The IM angle of all index patients was corrected from a preoperative mean of 13 degrees (range, 5 - 23) to a mean of 10 degrees (range, 5 - 21) postoperatively. Thirty-two patients (42\%) showed radiological malposition with valgus greater than 15 degrees, yet only two of these patients were symptomatic and had to be revised.

One patient suffered from rotational malposition in excessive supination which was corrected by a rotational osteotomy at the base of the first phalanx. An additional patient presented with a multiplanar malunion with excessive plantar flexion and pronation. This patient was treated by a revision of the fusion with plate and screw fixation.

Two patients required a medial sesamoidectomy. Both presented with hypertrophic sesamoids due to degeneration of the sesamoido-metatarsal complex and postoperative extension within the desired range. There were no cases of wound healing problems or infection. No hardware fractures were observed. No other complication occurred. Hardware removal was performed in twelve cases due to skin irritation and was not considered a revision.

\section{Discussion}

Arthrodesis of the MTP1 joint is generally a successful procedure, although historically, nonunion has been a major concern. However, the use of a dorsal locking plate and a metatarsophalangeal screw for MTP1 fusion has become the most commonly used fixation technique and has reduced the nonunion rate from $10 \%$ - $20 \%$ to $2 \%$ - $14 \%$ [3] [5]. While in the past most revision surgery after MTP1 fusion was performed due to nonunion, little is known about current rates and reasons for revision using modern implants. To the best of our knowledge, this study of 120 consecutive patients is the largest reported series providing such information and revealed that more reoperations were required for malposition (9\%) than for nonunion (3.3\%). This confirmed the findings of Kunovsky et al. [7] who in their recently published series of 56 MTP1 fusions using similar technology noted five percent revision for nonunion compared to nine percent for malposition.

While it seems obvious that correct positioning of the fusion is important for successful clinical outcomes [9], the relation between radiological positioning and related clinical symptoms leading to reoperation is not clear [10]. A number of recommendations for measuring and positioning of the great toe have been described [9] [11] [12] [13]. In this study the angle of the proximal phalanx in relation to the floor was used to describe the position in the sagittal plane [9] 
[13]. Unlike the more commonly used metatarsophalangeal angle, this angle does not depend on the slope of the metatarsal and remains the same in cavus or planus feet. However, both angles are dependent on the translation of the metatarsal head in the sagittal plane (elevatus). Thus, the required fusion angle cannot be expected to be the same in a patient with metatarsus primus elevatus as in a patient without; yet until now no specific recommendations for positioning of the toe in metatarsus primus elevatus exist in the literature.

The toe-to-floor angle can easily be reproduced during surgery by holding a board under the foot to simulate weight bearing [14] [15]. For positioning the MTP1 arthrodesis some surgeons rely on the distance of the tip of the toe the above mentioned board [6], and aim for $5 \mathrm{~mm}$ of clear space or a fingertip, but a recent study has shown that there is no statistical correlation between this distance and the postoperatively achieved angle [14]. This procedure is therefore not particularly reliable. Thus it has rather been recommended to allow for three centimeters of heel clearance with no extension in the IP joint when simulating push off [12], while still allowing for slight contact of the toe with the ground when standing. In patients with metatarsus primus elevatus this maneuver will likely result in a toe-to-floor angle of less than five degrees. Therefore, we aimed for a toe-to-floor angle in the sagittal plane between 0 and 15 degrees depending on the foot type.

In the sagittal plane, we found a mean postoperative dorsal extension of the toe in relation to the floor of 4.3 degrees (range, 0 - 14 degrees) and thus succeeded in positioning the toe within the desired range of the sagittal plane in all cases. The use of a precontoured plate may have contributed to this. Doty et al. [5] stated that the use of a precontoured plate with 10 degrees of extension and valgus consistently put them near the desired arthrodesis position of 15 - 25 degrees of dorsiflexion and 10 - 20 degrees of valgus. By using precontoured plates with 10 degrees of dorsal extension Goucher et al. [16] showed that the desired values could reliably be achieved. On the other hand, Mayer et al. [1] compared 102 MTP fusions with noncontoured plates to 26 arthrodesis with precontoured plates. They did not find significant differences in position, pain or complication rates and concluded that the use of precontoured plates did not seem to reduce malposition. In our study, despite presenting a radiological position within the desired range, some patients suffered clinically from malposition in the sagittal plane with insufficient extension and needed revision. We conclude that reaching the desired radiological angle seemed not to prevent revision surgery. There are two conceivable explanations. One, we may still not understand the true nature of the dynamic relation between position in the sagittal plane and the outcome, and/or two, the defined target range was inappropriate for the individual patient because the sagittal translation of the metatarsal head was not taken into consideration. More specific individual target ranges may need to be defined depending on the sagittal translation of the metatarsal head. Data analysis with scatterplot graphs did not allow for defining a target zone to avoid repositioning 
revision. Further studies will be needed to address this issue.

In the horizontal plane, valgus of 5 - 20 degrees is generally recommended. Fitzgerald [17] reported that a valgus of 20 degrees in the MTP1 joint reduced IP arthritis three fold. In our series, the position after the MTP1 arthrodesis in the 120 feet was found to be a mean valgus of 12 degrees (range, 0 - 31 degrees). No patient was fused in varus. Painful contact between the first and second toes accounted for most revisions due to malposition. Five patients needed repositioning by means of an Akin osteotomy. It is interesting to note that only two of them had a valgus of more than 15 degrees and were classified as radiological malposition. It is possible that the individual range of asymptomatic valgus depends on the width of the toes themselves and therefore a general recommendation may not be applicable. It is also possible that progressive reduction of the IM angle postoperatively is contributing to the problem since the intraoperative position may not be the one encountered at 6 months. The scatterplot analysis demonstrated that a target zone of 0 - 9 degrees of valgus would have avoided all repositioning revisions in the horizontal plane. A recommendation of $0-10$ degrees of valgus in the horizontal plane instead of the traditional 5 - 15 degrees may thus contribute to diminishing revision rates.

With revision as endpoint, this study does not provide information about patient satisfaction or function through scores. Furthermore, no measurement of the translation of the metatarsal head in the sagittal plane (elevatus) was made, which may explain why we have not been able to provide recommendation for MTP1 fusion angles. Future studies should include measurement of the translation of the metatarsal head in the sagittal plane (elevatus) in order to come up with matching values for positioning recommendation of MTP1 fusion in order to avoid revision for malposition.

\section{Conclusion}

The revision rate for nonunion after MTP1 fusion with the latest locking plate technology is low. Malposition is the leading cause for revision with a reoperation rate threefold higher than for nonunion. Excessive valgus and insufficient extension account for most malposition. Whereas a recommendation of $0-10$ degrees of valgus in the horizontal plane may contribute to diminishing revision rates, no conclusions could be drawn regarding an optimal position in the sagittal plane.

\section{Conflicts of Interest}

The authors declare no conflicts of interest regarding the publication of this paper.

\section{Disclosure}

One or more of the authors has received or will receive benefits for personal or professional use from a commercial party related directly or indirectly to the 
subject of this article: Lisca Drittenbass, MD, Mathieu Assal, MD, and Halah Kutaish MD report personal fees from Medartis and grants from Medartis during the conduct of the study.

The authors disclosed receipt of financial support for the research and institutional support, authorship, and/or publication of this article.

\section{References}

[1] Mayer, S.A., Zelenski, N.A., DeOrio, J.K., Easley, M.E. and Nunley II, J.A. (2014) A Comparison of Nonlocking Semitubular Plates and Precontoured Locking Plates for First Metatarsophalangeal Joint Arthrodesis. Foot and Ankle International, 35, 438-444. https://doi.org/10.1177/1071100714520695

[2] Politi, J., Hayes, J., Njus, G., Bennett, G.L. and Kay, D.B. (2003) First Metatarsal-Phalangeal Joint Arthrodesis: A Biomechanical Assessment of Stability. Foot and Ankle International, 24, 332-337. https://doi.org/10.1177/107110070302400405

[3] Hyer, C.F., Scott, R.T. and Swiatek, M. (2012) A Retrospective Comparison of Four Plate Constructs for First Metatarsophalangeal Joint Fusion: Static Plate, Static Plate with Lag Screw, Locked Plate, and Locked Plate with Lag Screw. The Journal of Foot and Ankle Surgery, 51, 285-287. https://doi.org/10.1053/j.jfas.2012.02.006

[4] Curtis, M.J., Myerson, M., Jinnah, R.H., Cox, Q.G.N. and Alexander, I. (1993) Arthrodesis of the First Metatarsophalangeal Joint: A Biomechanical Study of Internal Fixation Techniques. Foot and Ankle International, 14, 395-399. https://doi.org/10.1177/107110079301400705

[5] Doty, J., Coughlin, M., Hirose, C. and Kemp, T. (2013) Hallux Metatarsophalangeal Joint Arthrodesis with a Hybrid Locking Plate and a Plantar Neutralization Screw: A Prospective Study. Foot and Ankle International, 34, 1535-1540. https://doi.org/10.1177/1071100713494779

[6] Ellington, J.K., Jones, C.P., Cohen, B.E., et al. (2010) Review of 107 Hallux MTP Joint Arthrodesis Using Dome-Shaped Reamers and a Stainless-Steel Dorsal Plate. Foot and Ankle International, 31, 385-390. https://doi.org/10.3113/FAI.2010.0385

[7] Kunovský, R., Pink, T. and Jarošík, J. (2017) Arthrodesis of the First Metatarsophalangeal Joint by Locking Plate. Acta Chirurgiae Orthopaedicae Et Traumatologiae Cechoslovaca, 84, 453-461.

[8] Roukis, T.S. (2011) Nonunion after Arthrodesis of the First Metatarsal-Phalangeal Joint: A Systematic Review. The Journal of Foot and Ankle Surgery, 50, 710-713. https://doi.org/10.1053/j.jfas.2011.06.012

[9] Deland, J.T. and Williams, B.R. (2012) Surgical Management of Hallux Rigidus. Journal of the American Academy of Orthopaedic Surgeons, 20, 347-358. https://doi.org/10.5435/JAAOS-20-06-347

[10] Bayomy, A.F., Sangeorzan, B.J. and Ledoux, W.R. (2016) Arthrodesis of the First Metatarsophalangeal Joint: A Robotic Cadaver Study of the Dorsiflexion Angle. Journal of Bone and Joint Surgery, 92, 1754-1764.

[11] Alentorn-Geli, E., Gil, S., et al. (2013) Correlation of Dorsiflexion Angle and Plantar Pressure Following Arthrodesis of the First Metatarsophalangeal Joint. Foot and Ankle International, 34, 504-511. https://doi.org/10.1177/1071100713477386

[12] Kumar, S., Pradhan, R. and Rosenfeld, P.F. (2010) First Metatarsophalangeal Arthrodesis Using a Dorsal Plate and a Compression Screw. Foot and Ankle International, 31, 797-801. https://doi.org/10.3113/FAI.2010.0797

[13] Kelikian, A.S. (2005) Technical Considerations in Hallux Metatarsalphalangeal Arthrodesis. Foot and Ankle Clinics, 10, 167-190. 
https://doi.org/10.1016/j.fcl.2004.11.002

[14] Leaseburg, J.T., DeOrio, J.K. and Shapiro, S.A. (2009) Radiographic Correlation of Hallux MP Fusion Position and Plate Angle. Foot and Ankle International, 30, 873-876. https://doi.org/10.3113/FAI.2009.0873

[15] DeSandis, B., Pino, A., Levine, D.S., et al. (2016) Functional Outcomes following First Metatarsophalangeal Arthrodesis. Foot and Ankle International, 37, 715-721. https://doi.org/10.1177/1071100716642286

[16] Goucher, N.R. and Coughlin, M.J. (2006) Hallux Metatarsophalangeal Joint Arthrodesis Using Dome-Shaped Reamers and Dorsal Plate Fixation: A Prospective Study. Foot and Ankle International, 27, 869-876. https://doi.org/10.1177/107110070602701101

[17] Fitzgerald, J.A. (1969) A Review of Long-Term Results of Arthrodesis of the First Metatarso-Phalangeal Joint. The Journal of Bone and Joint Surgery, 51-B, 488-493. https://doi.org/10.1302/0301-620X.51B3.488 